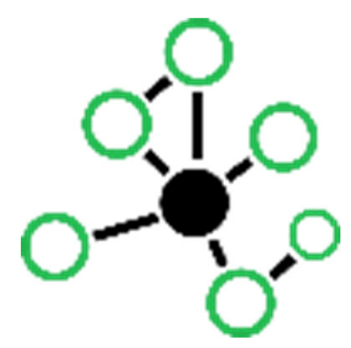

\title{
Towards Contagious Animal Disease Detection using Machine Learning
}

\author{
Boender, G.J. ${ }^{1}$, R. Petie ${ }^{1}$, A. Dekker ${ }^{1}$, J. Gonzales ${ }^{1}$ \\ ${ }^{1}$ Wageningen Bioveterinary Research, Wageningen University \& Research, Houtribweg 39, 8221 RA Lelystad, the \\ Netherlands \\ Corresponding author's e-mail: gertjan.boender@wur.nl
}

In livestock farms an increasing number of sensors is implemented to monitoring farm production and animal welfare, leading to large amounts of data. We investigated the potential of sensor data for the detection of contagious animal diseases, using machine learning for the interpretation of the data. An preliminary example of this is the classification of images of cows depending on their health status. The machine learning classification is performed with 25 images of cows before and 25 images after the Foot and Mouth infection for training purposes, while leaving the other for testing the performance of the trained network. This gave us a sensitivity and specificity of about $70 \%$. So, contagious animal disease detection using machine learning seems to be feasible, but many aspects need improvement before an in real life applicable digital diagnostic tool can be put forward. First, the format of the data for infected and susceptible animals should be uniformed to avoid classification based on other differences than the disease status. Furthermore, a larger training dataset is needed for further improvement of the performance of the machine learning. Finally, we wish to include data from other sensors, like sound and infra-red, as this would probably lead to more sensitive and more specific digital diagnostic test for contagious animal diseases. 